\title{
Kreislaufführung von Werkstoffen, Komponenten und Produkten: eine ökonomische Herausforderung
}

\begin{abstract}
Angesichts einer begrenzten Deponiekapazität der Erde und einer zunehmenden Kritikalität von Ressourcen und Lieferketten gewinnt die Kreislaufführung der Stoffflüsse zentrale Bedeutung. Der Abfall wird zum Wertstoff. Kenntnisse über seine stoffliche Zusammensetzung werden zum zentralen Ansatzpunkt der Steuerung umweltökonomisch effizienter Wertschöpfungskreisläufe. Durch die digitale Spiegelung der realen Prozesse wird dies möglich. Das Konzept des Total Design Management ermöglicht das simultane Optimieren von Werkstoff-, Produkt- und Recycling-Design. Nachhaltigkeit wird stringent in Bezug auf die durch den digitalen Raum gegebenen Systemgrenzen definiert. Aus volkwirtschaftlicher Sicht entsteht eine transaktionsökonomisch begründbare Abwägung zwischen internationaler Arbeitsteilung und Technologiesouveränität. Wie diese ausfällt, hängt stark von der Regulierung der Märkte und den Lieferkettenrisiken ab.
\end{abstract}

\begin{abstract}
Für das Steuern logistischer Ketten ist der Zugriff auf reale Stoffflüsse begleitende Informationen wichtig. Mit der Entwicklung moderner Kommunikationssysteme wurde es möglich, diese nicht nur parallel - mit der Fracht -, sondern bereits vorzeitig bereitzustellen, was ab Mitte der 1980er Jahre einen Schub hin zum Just in Time erzeugte. Von da an spielten Informationen in der Logistik eine wesentliche gestalterische Rolle für die stetige Rationalisierung der Wertschöpfungsprozesse. Auch durch das reale und virtuelle Prozesse integrierende Internet der Dinge (Industrie 4.0) und das Nutzen seiner Potenziale für geschlossen Stoffkreisläufe, werden sich Organisationsstrukturen nachhaltig ändern.
\end{abstract}

(C) Der/die Autor:in(nen) 2021. Open Access: Dieser Artikel wird unter der Creative Commons Namensnennung 4.0 International Lizenz veröffentlicht (creativecommons.org/licenses/by/4.0/deed.de).

Open Access wird durch die ZBW - Leibniz-Informationszentrum Wirtschaft gefördert.

* Der Verfasser dankt Manfred Füting für die intensive Diskussion beim Verfassen der Arbeit. Dem Ministerium für Wirtschaft, Wissenschaft und Digitalisierung des Landes Sachsen-Anhalt sei für die Förderung von Teilen dieser Forschung unter der Bewilligungsnummer I 108 gedankt.

Prof. Dr. Dr. h. c. Ulrich Blum ist Inhaber des Lehrstuhls für Wirtschaftspolitik und Wirtschaftsforschung an der Universität Halle-Wittenberg und Gründungsdirektor des Center for Economics of Materials (CEM) dort.
Abfall in einer techno-ökonomischen Sicht ist eine Ressource, auf die sich Geschäftsmodelle aufbauen lassen. In einem System geschlossener Stoffströme (Perey et al., 2018) spricht man von einem Post-Konsum und -Produktionswertstoff (PKPW). ${ }^{1}$ Für eine effiziente Kreislaufwirtschaft spielt die Digitalisierung eine zentrale Rolle, weil sie durch das Nutzen großer Mengen an relevanten Informationen die Grenzen menschlicher Prozesssteuerfähigkeit überwinden hilft (Kahneman und Tversky, 1979; Ettenson, Krogstad und Shanteau, 1987). Abbildung 1 zeigt diese kaskadenähnlichen Ablaufschritte der Aufbereitung (Wiederverwendung, Recycling oder Dekonstruktion), die teilweise der Umkehrung der in sogenannten Gozintographen dargestellten Produktionsschritte ähneln. Ziel ist es, eine möglichst hohe Qualität der aufbereiteten Stoffe bzw. Materialien unter Einbeziehung der Unwägbarkeiten der Zukunft zu erreichen (Blum und Boroskikh, 2021). Manche PKPW können direkt weitergenutzt werden, beispielsweise Hochleistungsmagnete, wenn ihre Geometrie - die Makrostruktur - dies ermöglicht; andere müssen erst zerlegt, im Extremfall mikrostrukturell aufbereitet werden. Nicht wiederverwertbare Wertstoffe können abschließend thermisch verwertet werden - bei Metallen durch Einschmelzen, bei kohlenstoffhaltigen Materialien durch Verbrennung, am besten Pyrolyse, wodurch Kohlenmonoxid entsteht, was wiederum eine Grundlage für

1 PKPW erscheint begrifflich schärfer als End-of-Life-Werkstoffe, weil es dieses „end of life“ nicht gibt. Darüber hinaus ist die Begrifflichkeit vor allem in Bezug auf den Export bedeutsam, denn Produkte können frei ausgeführt werden; gesetzliche Ausnahmen bestehen gemäß AuBenwirtschaftsgesetz nur in sicherheitsrelevanten Bereichen. Abfall hingegen ist genehmigungspflichtig. 
Abbildung 1

Grundmodell der Aufbereitung

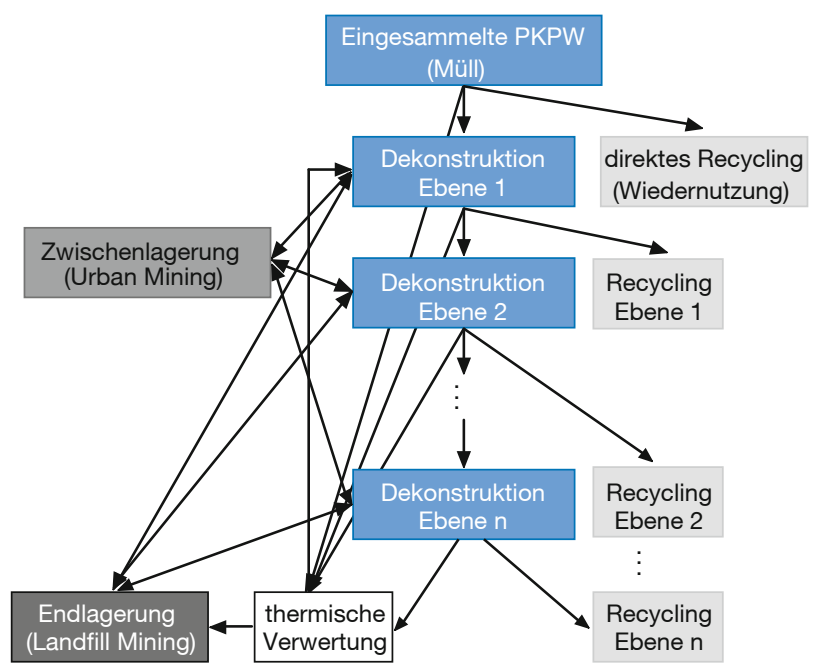

Quelle: eigene Darstellung.

Industriegase sein kann. ${ }^{2}$ PKPW-Lager können für spätere Verwertungen angelegt werden. Im Fall von vorhandenen Deponien spricht man beim Extrahieren von Wertstoffen vom Landfill Mining; eine spezielle Form ist das Aufschließen alter Bergbauhalden, das Re-Mining (Büttner und Gutzmer, 2017). Urban Mining, Landfill Mining und Re-Mining können auch strategisch interpretiert werden: Es wird für die Zukunft gelagert bzw. deponiert, weil eine Erwartung in verbesserte neue Technologien besteht; man könnte dann von Warehouse Mining sprechen. Bei elektronischen Geräten wie Smartphones oder Tablets dürfte eine Zwischenlagerung beim aktuellen Stand der Technik (noch) sinnvoll sein. ${ }^{3}$ Bei idealer Aufbereitung läge der Eintrag in die Endlagerung nahe null. ${ }^{4}$

Hier wird ein innovatives Modell einer Kreislaufwirtschaft vorgestellt, in dem die drei zentralen Stufen des Designs, das Werkstoffdesign ${ }^{5}$, das Produktdesign und das Design für Dekonstruktion und Recycling, auch Design for Environment genannt (Low et al., 2014), als simultanes Optimierungssystem formuliert werden. Dies geht über

2 Dies könnte der Verbrennung überlegen sein, sobald hochpreisige $\mathrm{CO}_{2}$-Lizenzen verpflichtend werden (IMWS, 2018). Über den Umweg der Pyrolyse und damit der Gase lassen sich hochkomplexe Kunststoffe preiswerter produzieren, als dies z. B. durch Rezyklate auf Basis von Lösungsverfahren möglich ist.

3 Das Wuppertal Institut (2013) schätzt den stofflichen Wert eines Smartphones oder Tablets bei etwa 1 bis 2 Euro pro Gerät.

4 Cossu und Williams (2015) geben eine Kaskade an, deren äußerer Ring die zirkuläre Wirtschaft ist; in diese eingebettet ist das Vermindern von PKPW, in diesem wiederum die Wiederverwertung und unterhalb dieser dann Urban Mining und Landfill Mining.

5 In Deutschland baut rund $70 \%$ der Innovationsleistung der Industrie auf neuen Werkstoffen auf (acatech, 2007; VDI, 2014). die bisherigen Ansätze hinaus (Bocken et al., 2016), weil es gleichzeitig den Lebenszyklus gemäß der zirkulären Ökonomie schließt und dabei vom Primat des reinen Produktdesigns abrückt und das Werkstoff- und das Recyclingdesign einbezieht (Dietz, 2021).

Am Beispiel des Stoffflusses eines Magneten wird auf solche Informationen verwiesen, welche die Herstellungsund abschließenden Verwertungsschritte begleiten. Dabei zeigt sich, dass nicht alle Komponenten, die in das Endprodukt eingehen, im Knappheitssinn gleichbedeutend sind; einige können Engpasscharakter besitzen, weshalb auf die Kritikalität von Rohstoffen eingegangen wird, die einen zunehmenden Zwang zur Wiederverwertung erzeugt. Damit wird auch das Erfordernis verdeutlicht, das Leben eines Werkstoffs - bis zu seiner Verwertung - möglichst detailliert zu erfassen, um den Kreislauf effizient zu schließen, was den Anspruch auf ganzheitliches Design verwirklichen hilft. Dies kann wiederum die Technologiesouveränität bei kritischen Versorgungslagen im Sinn eines „Kampfes um Wertschöpfungsketten“ (Blum, 2018) erhöhen. ${ }^{6}$

\section{Stofffluss, digitaler Zwilling und Design}

Die Netzwerkstrukturen einer Kreislaufführung beeinflussen die Grenze zwischen Markt und Unternehmen (Coase, 1937; Williamson, 1985, 2002) und somit die dadurch verbundenen Informationsasymmetrien erheblich. Zusätzlich verstärken die mehrstufig organisierten Designansprüche das Kontrollproblem zwischen Prinzipalen und Agenten: Agenten sollen die Prinzipale nicht hintergehen. Die digitale Spiegelung der physischen Abläufe hilft, um, wie von der Europäischen Union gefordert, das Downcycling zu verhindern (EU, 2020), Situationen der moralischen Versuchung und der Hold-ups zu vermeiden und durch Signalisieren hoher Qualität Reputation zu signalisieren - alles zwingend notwendige Voraussetzungen für eine effiziente Kreislaufführung. Infolge einer Vielzahl von Informationsasymmetrien entlang der Wertschöpfungskette ergibt sich stets ein Abwägen alternativer institutioneller Arrangements infolge von Transaktionskosten im Informations- ebenso wie im realen Bereich.

Abstimmungsprobleme im Wertschöpfungsprozess und damit in Wertschöpfungsketten bzw. -netzwerken entstehen bereits dadurch, dass der Weg von der Gewinnung, beispielsweise in einer Mine, über Anreicherung, Herstellen funktionaler Materialien, der Nutzung, schließlich der PKPWVerwertung keinesfalls linear ist, was zu einer digitalen Erfas-

6 Als Technologiesouveränität ist die Fähigkeit definiert, kritische Technologien und Ressourcen als Unternehmen, Staat oder Staatengruppe (z. B. EU) vor Ort im Zugriff zu haben. Offensichtlich ist dies in erheblichem Maß mit einer qualifizierten Risikoabschätzung verbunden, d. h. die entsprechenden Transaktionskosten spielen die entscheidende Rolle beim Abwägen zwischen Fremdbezug oder Eigenfertigung. 
Abbildung 2

Grundmodell des digitalen Verzwillingens

Reale Welt

Theorie und Modelle

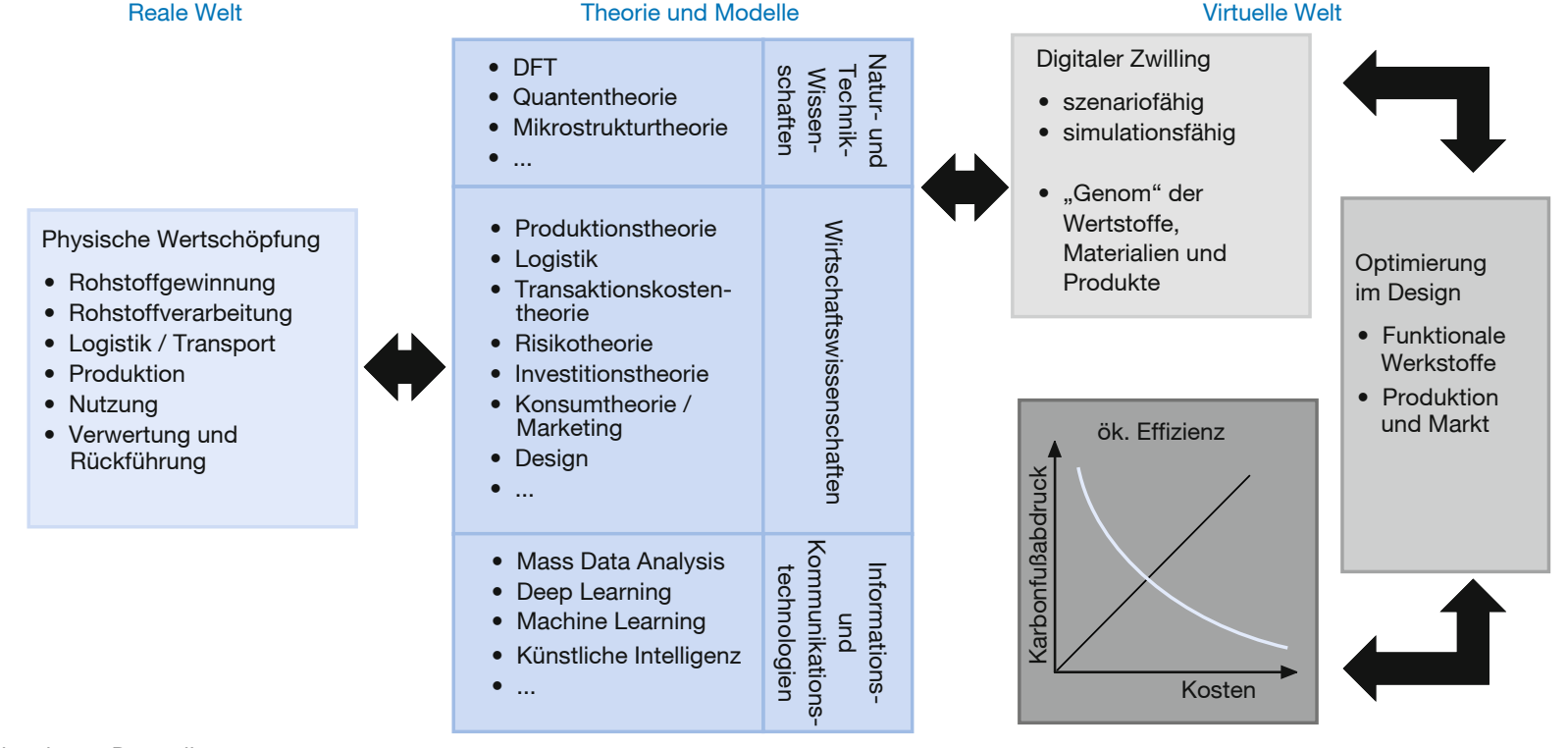

Quelle: eigene Darstellung.

sung einlädt, um das Entstehen von Informationsasymmetrien zu verhindern. Bei der Gewinnung ist Koppelextraktion die Normalität, beispielsweise Indium-Zink, Rhenium-Koltan (Kobalt)-Kupfer-Nickel oder Neodym-Lanthan-Cer. ${ }^{7}$ Funktionale Materialen benötigen bestimmte Trägerwerkstoffe, z. B. Eisen oder Aluminium für Magnete; auch deren Engpass kann Nutzungsstränge massiv unter Druck setzen (Reuter et al., 2018). Die Aufbereitung wiederum hängt wesentlich davon ab, welche Legierung mit welchen speziellen Gefügeparametern und welchen Verfahren geschaffen wurde. Damit können sowohl im Gewinnungs- wie im Produktions- und Verwertungsprozess wesentliche Engpässe auftreten.

Das Internet der Dinge, also Industrie 4.0, das hier dem physischen Stofffluss einen digitalen Zwilling zur Seite stellt, wodurch eine industrielle Symbiose hergestellt wird, gewinnt damit an Stellenwert. Insbesondere die sogenannten 3R (reduce, reuse, recycle) können damit aus ganzheitlicher Perspektive angegangen werden (Tseng et al., 2018). Dieser Zwilling, der als simulations- und szenariofähiges Pendant zum realen System gedacht ist (Schleich et al., 2017), gewinnt bei der dynamischen Betrachtung eine Biografieähnlichkeit, weil seine wesentlichen Daten über Herkunft und Produktionsschritte erfasst und verarbeitet werden, also der Weg von den Roh- und Ausgangsstoffen über die Werkstoffe, die Halbzeuge und Produkte bis schließlich zu den zurückzuführenden PKPW. Schleich et al. (2017) folgend sind die wesentlichen Eigen-

7 Mit einem $\mathrm{kg}$ Neodym werden zugleich rund 1,5 kg Lanthan und etwa 2,5 kg Cer gefördert. schaften eines derartigen Modells seine Ähnlichkeit und Darstellungstreue, seine Erweiterbarkeit, seine Interoperabilität und seine Skalierbarkeit. Das digitale Verzwillingen erfolgt dadurch, dass gleichermaßen am realen und am digitalen Körper physikalische und digitale Operationen entlang seiner Nano-, Mikro- oder Makrostruktur durchgeführt werden können (Fraunhofer-Verbund Materials).

Abbildung 2 zeigt Verzwillingen des Realen mit dem Virtuellen. Es entsteht eine möglicherweise marktmächtige digitale Plattform (Ramakrsihna et al., 2018). Die erforderlichen Informationen im digitalen Zwilling lassen sich auch als Genom bezeichnen (Wang et al., 2014; Rajan 2015). Dabei kann die Bewertung in vielen Fällen monetär erfolgen, in anderen Fällen bietet sich der Karbonfußabdruck an, wie Low, Lu und Song (2014) zeigen. Durch eine konvexe Isoquante wird rechts unten im Diagramm die Preis-Mengen-Austauschbeziehung als Knappheitskalkül einbezogen, und der Expansionspfad zeigt das Verhältnis aus monetären und nichtmonetären Kosten, beispielsweise auf Basis von Umweltlizenzen. Die Isoquante stellt zugleich die Grenze dar, jenseits derer eine Lebenszyklus-Optimierung nicht sinnvoll ist.

\section{Der Makro-Stofffluss im Beispiel}

Tabelle 1 zeigt die mögliche Biografie für einen Hochleistungsmagneten auf Basis von Neodym, Dysprosium, Bor und Eisen, die demzufolge den Kern des digitalen Genoms in ihren stofflichen Eigenschaften darstellen. Das Neodym kommt von Baotou Steel aus China und wird von COSCO über die österreichische Firma Treibacher nach Deutsch- 
Tabelle 1

Beispiel für die Grobstrukturen einer Biografie (Magnet)

\begin{tabular}{|c|c|c|c|c|c|}
\hline & $\begin{array}{l}\text { Herkunft des Roh- } \\
\text { bzw. Werkstoffs }\end{array}$ & Halbzeug & Fertigprodukt & Verwertung & Empfänger Rezyklat \\
\hline Region & $\begin{array}{l}\text { Länder, Minen (Chi- } \\
\text { na, Baotou Steel) }\end{array}$ & $\begin{array}{l}\text { Länder, Unternehmen } \\
\text { (Deutschland Vaku- } \\
\text { umschmelze) }\end{array}$ & $\begin{array}{l}\text { Länder, Unternehmen } \\
\text { (OEM) (Deutschland, } \\
\text { Brose, VW) }\end{array}$ & $\begin{array}{l}\text { Länder, Unternehmen } \\
\text { (OEM) (Deutschland, Re- } \\
\text { mondis; Belgien: Umicor) }\end{array}$ & $\begin{array}{l}\text { Länder, Unternehmen } \\
\text { (Deutschland Vakuum- } \\
\text { schmelze; Deutsch- } \\
\text { land, Brose) }\end{array}$ \\
\hline $\begin{array}{l}\text { Internationale und } \\
\text { betriebliche Logistik }\end{array}$ & $\begin{array}{l}\text { Verantwortlichkeit für } \\
\text { die Transportkette } \\
\text { (China: COSCO; Ös- } \\
\text { terreich: Treibacher) }\end{array}$ & $\begin{array}{l}\text { Verantwortlichkeit } \\
\text { für die Transport- } \\
\text { kette (Deutschland: } \\
\text { Schenker) }\end{array}$ & $\begin{array}{l}\text { Verantwortlichkeit für die } \\
\text { Wertschöpfungs-und } \\
\text { Transportkette (Deutsch- } \\
\text { land: Brose, VW, Schenker) }\end{array}$ & $\begin{array}{l}\text { Verantwortlichkeit für die } \\
\text { PKPW-Kette (Deutsch- } \\
\text { land: Remondis; Belgien: } \\
\text { Umicor) }\end{array}$ & $\begin{array}{l}\text { Künftige Wert- } \\
\text { schöpfungsketten, } \\
\text { Importe, Exporte }\end{array}$ \\
\hline $\begin{array}{l}\text { Verarbeitungs- } \\
\text { technologie }\end{array}$ & $\begin{array}{l}\text { Makro-, Mikrostruk- } \\
\text { turen }\end{array}$ & $\begin{array}{l}\text { Ausgangsmateria- } \\
\text { lien, Legierungen, } \\
\text { Makro-, Mikro-, } \\
\text { Nanostrukturen }\end{array}$ & $\begin{array}{l}\text { Ausgangsmaterialien, Le- } \\
\text { gierungen, Makro-, Mikro-, } \\
\text { Nanostrukturen }\end{array}$ & $\begin{array}{l}\text { Wiedernutzung, Dekon- } \\
\text { struktion, Recycling, } \\
\text { Technologien }\end{array}$ & \\
\hline Standards & $\begin{array}{l}\text { Normen und Zertifi- } \\
\text { zierungen von Betrie- } \\
\text { ben und Produkti- } \\
\text { onen, z. B. ISO } 9000 \mathrm{ff}\end{array}$ & $\begin{array}{l}\text { Normen und Zertifi- } \\
\text { zierungen von Betrie- } \\
\text { ben und Produkti- } \\
\text { onen, z. B. ISO } 9000 \mathrm{ff}\end{array}$ & $\begin{array}{l}\text { Normen und Zertifizierun- } \\
\text { gen von Betrieben und } \\
\text { Produkten }\end{array}$ & $\begin{array}{l}\text { Normen und Zertifizierun- } \\
\text { gen von Betrieben und } \\
\text { Produkten }\end{array}$ & $\begin{array}{l}\text { In Bezug auf die Quali- } \\
\text { tät der wiederverwen- } \\
\text { deten Magnete z. B. } \\
\text { DIN EN 10330:2015-11 }\end{array}$ \\
\hline $\begin{array}{l}\text { Post Konsum- und } \\
\text { Produktionsprozesse }\end{array}$ & & & & $\begin{array}{l}\text { Verbrennung, Verschwelung, } \\
\text { Rezyklieren, Endlagerung }\end{array}$ & \\
\hline Daten & $\begin{array}{l}\text { Digitaler Zwilling auf } \\
\text { Basis des Materials } \\
\text { Data Space (MDS } ®) \text {, } \\
\text { d.h. Biografie }\end{array}$ & $\begin{array}{l}\text { Digitaler Zwilling auf } \\
\text { Basis des Materials } \\
\text { Data Space (MDS } ®) \text {, } \\
\text { d.h. Biografie }\end{array}$ & $\begin{array}{l}\text { Digitaler Zwilling auf Basis } \\
\text { des Materials Data Space } \\
\text { (MDS } ®), \text { d. h. Biografie, } \\
\text { insbesondere Technolo- } \\
\text { giedaten }\end{array}$ & $\begin{array}{l}\text { Digitaler Zwilling auf Basis } \\
\text { des Materials Data Space } \\
\text { (MDS } \otimes) \text {, d.h. Biografie, } \\
\text { insbesondere Technolo- } \\
\text { giedaten }\end{array}$ & $\begin{array}{l}\text { Abgleichen der Daten } \\
\text { zwischen der Biografie } \\
\text { und den Anforderun- } \\
\text { gen }\end{array}$ \\
\hline
\end{tabular}

Quelle: eigene Darstellung.

land an die Vakuumschmelze geliefert, die daraus einen Neodym-Dysprosium-Bor-Hochleistungsmagneten (NdDyFeB-Magnet) für die Autoindustrie fertigt, der bei Brose in ein Sekundäraggregat für einen $\mathrm{PkW}$ für $\mathrm{VW}$ verbaut wird. ${ }^{8}$ Analog kann die Kette für andere Werkstoffe aufgebaut werden, also bei dem besagten Magneten wären das Bor, Eisen oder Dysprosium, bei anderen Magneten Nickel, Samarium und Kobalt sowie Aluminium, usw. Am Ende seiner Nutzung kann der Magnet entweder von der Stahlindustrie - quasi als Beigabe - eingeschmolzen, durch Versprödung mit Wasserstoff in seine Bestandteile zerlegt, die dann rezykliert werden oder als Ganzes, gegebenenfalls nach Neumagnetisierung, erneut in einer Autokomponente verbaut werden. $\mathrm{Da}$ die Kombinationen der Bestandteile nicht beliebig sind, kann das Fehlen einzelner Komponenten die gesamte Fertigung aus den Fugen geraten lassen. Dies geschah beispielsweise bei der Seltene-Erde-Krise 2010. Dann sind Substitute gefragt. So gerieten die in den 1960er Jahren gängigsten Hochleistungsmagnete auf der Basis von Aluminium, Nickel und Kobalt (AINiCo) bzw. Kobalt und Samarium (SmCo) durch die Kongokrise unter Druck, was die Entwicklung neuer Hochleistungsmagnete auf der Basis Seltener Erden beschleunigte. ${ }^{9}$

8 Die angesprochenen Unternehmen sind in den entsprechenden Geschäftsfeldern aktiv, die Geschäftsbeziehungen fiktiv.

9 Kritische Ressourcen gerieten regelmäßig unter strategischen Druck im Rahmen von Wirtschaftskriegen (Blum, 2020).
Standards bilden eine wesentliche Voraussetzung für das effektive und effiziente Verzwillingen realer und virtueller Welten. Wer die Norm hat, kontrolliert den Markt (Blum, Bahke und Eickhoff, 2002), auch in digitalen Beziehungen. Wichtige Kenngrößen und ihre Messung werden durch Normen festgelegt. Für Magneten sind das beispielsweise die Koerzitivfeldstärke oder die Temperaturstabilität. Neben der Makrostruktur, also der Geometrie, die für den Einbau wichtig ist, sind weitere Parameter aus der Mikrostruktur (Gitterstruktur, Grenzflächen) und der Nanostruktur (Atomanordnung) für den Einsatz wichtig. Diese Daten erleichtern es dem Verwertungsunternehmen, beispielsweise Remondis in Deutschland für das Sammeln oder Umipor in Belgien für das Aufarbeiten, zu entscheiden, auf welcher der möglichen Verwertungsebenen rezykliert werden soll.

\section{Kritikalität als Verwertungstreiber}

Der Begriff der Kritikalität wird im ökonomischen Umfeld vor allem auf Roh- und Werkstoffe sowie auf Technologien bezogen und speist sich aus zwei Quellen.

- Zur ersten Dimension zählt die Verfügbarkeit und damit auch die Vulnerabilität. Sichtbar werden diese durch Preisspitzen, eine Verknappung, möglicherweise auch eine Nichtverfügbarkeit. Heute treten die Umweltfolgen der bergmännischen Extraktion, der Anreicherung, Verarbeitung und Entsorgung der Endprodukte am Ende des Le- 
benszyklus hinzu. Typische Probleme bei der Förderung Seltener Erden sind beispielsweise die Radioaktivität der Vorkommen, die die Gewinnung mit hohen Kosten belastet. ${ }^{10}$ Eigentlich sollten sich Verfügbarkeit, Vulnerabilität und Umweltkosten in (Knappheits-)Preisen widerspiegeln; dieser Ausweis erfolgt nur in begrenztem Rahmen, häufig asymmetrisch und schockartig - im Extremfall: Nichtverfügbarkeit, also Preis unendlich! Kobaltknappheit trifft Hersteller von Magneten auf Basis von Samarium oder Aluminium und Nickel. Geraten sie, wie anlässlich der Kongokrise vor Jahrzehnten, unter Druck, entstehen Substitute auf Basis von Neodym und Dysprosium. Diese kamen in Not anlässlich der Seltene-Erden-Krise 2010.11 Begünstigt sind dann die Anwender von Elektromagnettechnologien. Deren Achillesferse ist jedoch der Kupferpreis. Das strategische Nutzen dieses Wechselspiels kann sich zu extremen Bedrohungen entwickeln. ${ }^{12}$

- Im technischen Kontext hat der Begriff Kritikalität unter anderem Wurzeln in der Nuklearindustrie und in der Thermodynamik. Übertragen auf wirtschaftliche Zusammenhänge sind solche Ressourcen als kritisch zu bezeichnen, die in einem sehr allgemeinen Sinne sicherheitspolitisch bedeutend und für den laufenden Betrieb der Wirtschaft erforderlich sind. Leichte Störungen lösen dann massive Engpässe aus. Ein Innovator, der ein Substitut entwickelt, kann diesen Engpass beseitigen und zum „industriellen Führer" werden, der den Produktlebenszyklus festlegt.

Schon die Bewältigung einer globalen Energiewende erfordert Wachstumsraten bei der Förderung ganz gewöhnlicher Metalle, die als kritisch gelten müssen (Vidal, Goffé und Arndt, 2013). Da viele hochspezifische Rohstoffe nur als Koppelprodukte vorkommen, ist ihre Gewinnung an die großen Ausbeutungen der hauptsächlichen Metalle gekoppelt. So ist Indium ein Nebenprodukt der Zinkerze, Rhenium und Kobalt sind Nebenprodukte des Kupfers, dessen Förderung um das Jahr 2040 ihren Höhepunkt erreichen wird (Kerr, 2015). Koppelextraktion erzeugt einen ergänzenden Druck, die überschüssige Ressource sinnvoll zu nutzen, gelegentlich ergeben sich Optionen für eine

10 So wurde die US-amerikanische Mine Mountain Pass unter anderem aus diesen Gründen unwirtschaftlich und ging bereits zweimal in Insolvenz, was China zum dominanten Anbieter aufsteigen ließ.

11 Tesla verbaut erst in den neuen (kleinen) Modellen Permanentmagnete; frühere, große Modelle besitzen Drehstrom-Asynchron-Elektromotoren, weil diese wegen der Wicklungen etwa ein Drittel mehr Platz benötigen.

12 Auf einer Gedenktafel im Museum des Baotou Research Institute for Rare Earths (BRIRE) findet sich folgendes Zitat von Deng Xiaoping 1992: „Es gibt Öl im Nahen Osten, es gibt Seltene Erden in China. Die Seltenerdressourcen in China machen $80 \%$ der bekannten globalen Reserven aus. Die Position der Seltenen Erden in China ist vergleichbar mit der Rolle des Öls im Nahen Osten. Seltene Erden haben eine wichtige strategische Bedeutung. Wir müssen die Seltenerdindustrie gut entwickeln und Chinas Stärke in Bezug auf Seltenerdressourcen nutzen.“
Substitution. So sind für die Elektromobilität die Seltenen Erden Neodym und Dysprosium beim Bau von Hochleistungsmagneten entscheidend - letzteres kann sich in erheblichem $\mathrm{Maß}$ zu einem limitierenden Faktor entwickeln (Hoenderdaal et al., 2013). Das im Periodensystem neben dem Neodym stehende Cerium wird meist gemeinsam mit diesem und Lanthan gewonnen, weshalb das partielle Ersetzen von Neodym durch Cerium vorteilhaft ist (Wehrspohn, Drossel und Stauber, 2018), und das verfügbare Lanthan könnte als Träger zur Speicherung von Wasserstoff ökonomisch sinnvoll eingesetzt werden.

Die Aufbereitung mindert bei kritischen Rohstoffen den externen Bedarf, machen ein Land möglicherweise weniger rohstoffabhängig. Blum (2018) hat gezeigt, dass China diesen Weg einer relativen - nicht absoluten - Deglobalisierung sowohl rohstofftechnisch als auch technologisch beschreitet und dies seit dem Herbst 2020 im Konzept der Zwei Wirtschaftskreisläufe - einem nationalen, einem internationalen intensiviert. Von den wichtigen Wirtschaftsnationen der Welt ist China die einzige, die in den vergangenen Jahrzehnten ihre Wertschöpfungstiefe - und damit auch ihre Technologiesouveränität - erhöhen konnte. Die Aufbereitungskosten sind produktabhängig, weshalb im Folgenden die Kostenstruktur eines einzelnen Aufbereitungsvorgangs betrachtet wird. Da dieser im monopolistischen Wettbewerb mit anderen Aufbereitungen und gegebenenfalls stofflichen Substituten steht, bieten sich die Durchschnittskosten als Angebotsreferenz an (Greenhut, Norman und Hung, 1987). Die angelieferten bzw. nachgefragten Aufbereitungsmengen sind zufallsabhängig und folgen aus Gründen der Einfachheit einer Dreieckswahrscheinlichkeitsverteilung. Die Mengenangebote bestimmen die Breite eines Blocks, dessen Höhe vom Durchschnittskostenminimum bei optimaler Ausbringungsmenge bis hin zu den höchsten Durchschnittskosten der Aufbereitung im relevanten Mengenbereich abhängt. Abbildung 3 zeigt den Verlauf einer Durchschnittskostenfunktion (rechte Skala) und die dem Mengenplan zugrunde liegenden Eintrittswahrscheinlichkeiten auf Basis der Dreiecksverteilung (linke Skala). Auf dieser Grundlage lässt sich ein Erwartungswert der aufzubereitenden Menge ableiten, die hier bei etwa acht Stück liegt, weshalb das Unternehmen tendenziell oberhalb des effizienten Durchschnittskostenminimums anbietet. Unternehmen werden versuchen, die Technologie, also das Durchschnittskostenminimum, dem Erwartungswert auf Grundlage der zugrunde liegenden Risikoverteilung anzupassen, um möglichst hohe Effizienzgewinne - gerade auch gegenüber der Konkurrenz - zu erzielen.

Auf dieser Basis lässt sich ein Kostenblock ausweisen, dessen horizontale Dimension die Menge und deren vertikale Dimension die Kosten sind. Unterster Kostenwert ist das Durchschnittskostenminimum, oberster sind die Kosten entlang der Durchschnittskostenfunktion mit dem 
Abbildung 3

Erwartete Durchschnittskosten im Merit-Order-Modell

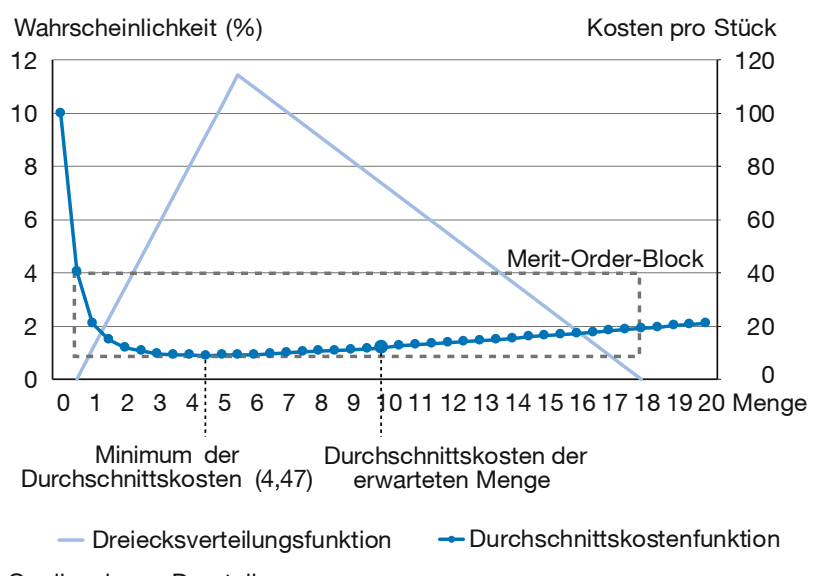

Quelle: eigene Darstellung.

höchsten Wert entlang der Mengen unterhalb der Verteilungsfunktion. ${ }^{13}$ Diese Blöcke lassen sich nach steigenden Erwartungswerten anordnen. Es sei dabei davon ausgegangen, dass die Kosten der Wiederverwendung, der Dekonstruktion bzw. des Rezyklierens von der ursprünglichen Verwendung abhängen, beispielsweise von einer Seltenen Erde in dem einen oder anderen Magneten, in Kondensatoren, Leuchten usw. Daraus entsteht eine Günstigkeitsreihenfolge (Merit-Order) der einzelnen Aufbereitungen entsprechend einem steigenden Aufwand, woraus die Angebotsfunktion abgeleitet wird..$^{14}$ Deren Position und Steigung ist von den Aufbereitungstechnologien der Werkstoffe in den jeweiligen Verwendungen abhängig, also von technologischen Parametern, aber auch von der geforderten Reinheit des Rezyklats, usw. Dies wird in der Abbildung 4 gezeigt. Ergänzt wird dieses Angebot, das die Kosten der jeweiligen erwarteten Mengen verbindet, durch die Nachfrage nach Rohstoffen aus Rezyklaten. Aus den damit abzuleitenden Marktbedingungen folgt die mögliche Kritikalität, also kritische strategische Verfügbarkeit.

Die Nachfragebereitschaft ist auch ein Ergebnis der Marktverhältnisse bei den originären Ressourcen - hier Neodym -, durch die dann die entsprechenden Aufbereitungsangebote mobilisiert werden. Im konkreten Fall werden fünf Ver-

13 Auf Basis einer Kostenfunktion $K=K f+20^{*} x+x 2$ ergibt sich ein Block mit Mengenintervall $(0,5 / 18)$ und Kostenintervall $(9,94 / 40,5)$.

14 Dies unterscheidet die Günstigkeitsfolge von der Merit Order in den Energiemärkten, bei denen die Anordnung nach Grenzkosten erfolgt. Verbunden mit der Einspeisungsverpflichtung regenerativer Energien mit niedrigen Grenzkosten führt dies zu einer preisdämpfenden Wirkung an Spotmärkten (Sensfuß, Ragwitz und Genoese, 2008; Cludius et al., 2014). Dies könnte auch für Rohstoffe gelten, wenn für Aufgearbeitetes eine Verarbeitungspriorität bestünde. Auch könnten die Fixkosten bei dezentralen Aufbereitungstechnologien anteilig niedriger liegen als bei der Exkavation.
Abbildung 4

Günstigkeitsfolge (Merit Order) und Kritikalität

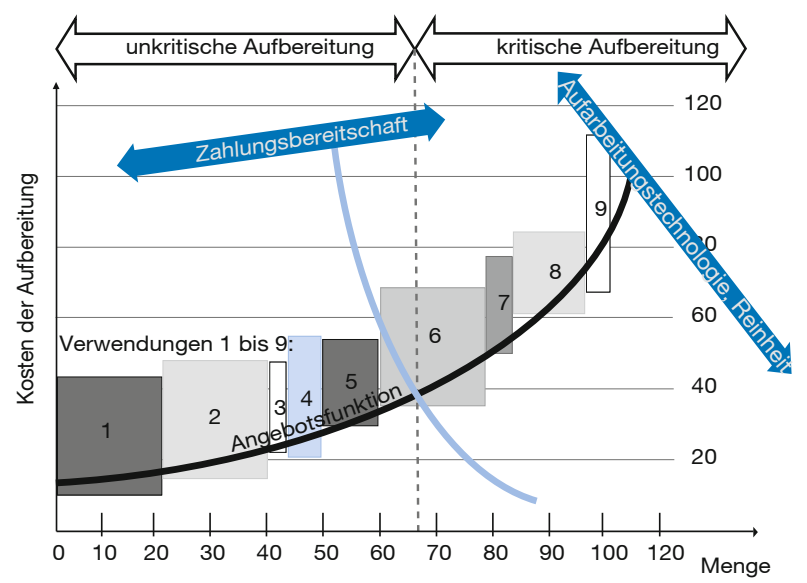

Quelle: eigene Darstellung.

wendungen des Neodyms herangezogen, z. B. Magnete aus Windgeneratoren, Elektromotoren von Pkw, E-Rollern, E-Bikes und Nabenmotoren von Schienenfahrzeugen; die Aufbereitung der sechsten Verwendung - z. B. in Lautsprechern - ist marginal; alle übrigen Bereiche sind zu teuer, beispielsweise Ohrhörer von Smartphones, vor allem wegen der Kosten für das Einsammeln kleinster Mengen. Damit lässt sich in Abbildung 4 ein kritischer von einem unkritischen Bereich in der Aufbereitung unterscheiden.

\section{Total Design Management}

Ein effizientes Lebenszyklusmanagement muss die Werkstoffzusammensetzungen so beeinflussen, dass sie gleichermaßen den Anforderungen des Marktes und der Aufbereitung genügen. In Abbildung 5 erfolgt die Abstimmung der unterschiedlichen Designstufen nach dem Marginalkalkül: So müssen die zusätzlichen Kosten eines aufbereitungsfreundlichen Designs bei konstanter Wertschätzung am Markt mindestens von den Ersparnissen eines späteren - aber unsicheren - Recyclings oder einer Dekonstruktion aufgewogen werden. Total Design Management (TDM) bedeutet eine Optimierung mit Blick auf die Kosten und die in Geld bewerteten ökologischen Fußabdrücke auf Basis der durch den digitalen Zwilling gesetzten Systemgrenzen. Das Informationsmanagement des Aufbereitungsunternehmens wird zum wirtschaftlichen Mittler und erhält damit eine starke Marktposition im Sinne einer Plattform.

Das Grundmodell des TDM findet sich in Abbildung 6. Die drei wesentlichen Stufen des Designprozesses sind entsprechend dem Stofffluss lokalisiert, der sich in einem Kreislauf bewegt. Aus diesem gibt es temporäre bzw. dauerhafte Ausgänge zur Lagerung bzw. finalen Entsorgung. Das Kalkül des simultanen Optimierens steht unter 


\section{Abbildung 5}

Das Ausgangsmodell der funktionalen Werkstoffe

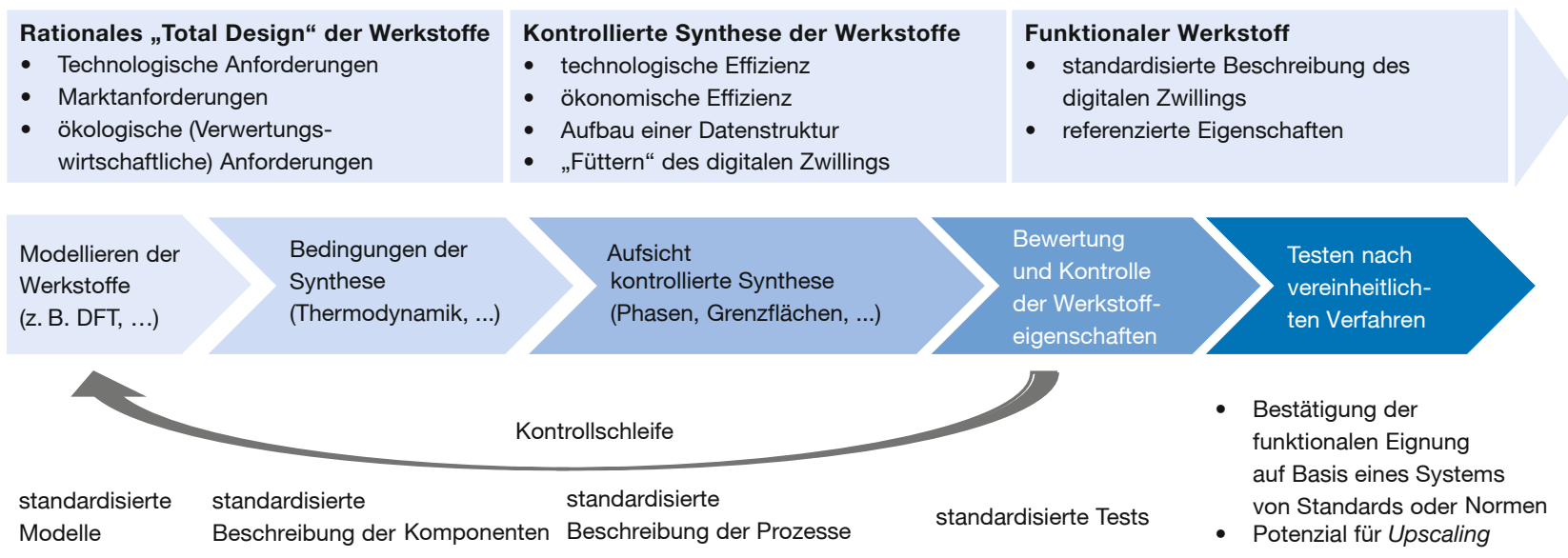

Quelle: eigene Darstellung.

einem wichtigen Zeitvorbehalt, weil nicht klar ist, ob das verwertungsfähige bzw. wiederaufbereitete Produkt in der Zukunft noch benötigt wird oder ob künftig verbesserte, also kostengünstigere Aufbereitungstechnologien zur Verfügung stehen als derzeit. Bei Produkten mit einer zeitlich kurzen Verweildauer im Markt, beispielsweise Verpackungskunststoffen, kann das gegenwärtige funktionale Design mit den Möglichkeiten der Dekonstruktionsbzw. Recyclingmethoden direkt abgestimmt werden. Vor allem bei langlebigen Gütern erleichtert ein digitaler Zwilling die Entscheidungsfindung mittels Szenariobildung und der Realoptionen. ${ }^{15}$

15 Realinvestitionen verwandeln hochflexibles Kapital in feste Anlagen - im Extremfall werden Kosten versenkt. Deren Inflexibilität sendet wiederum starke Signale an die Konkurrenz, sodass aus dieser anlagenbedingten Inflexibilität durchaus strategische Flexibilität im Markt entsteht. Die entsprechenden Erträge und Risiken werden gegenübergestellt.

\section{Abbildung 6}

Grundmodell des Total Design Management

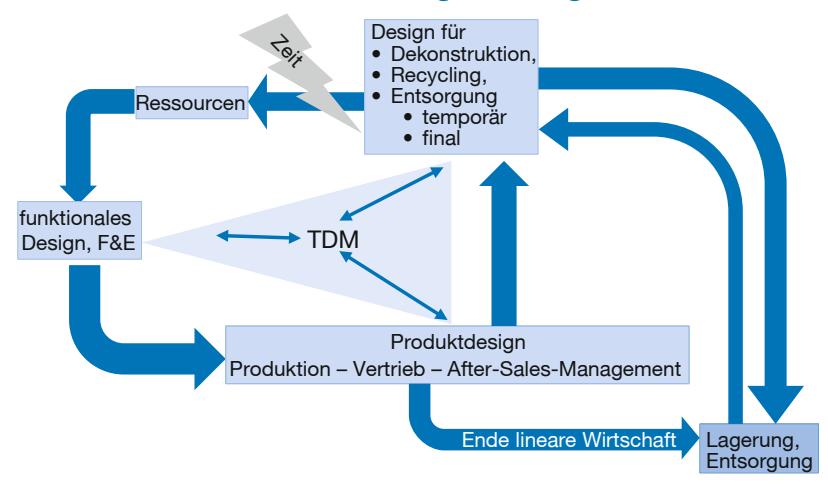

Quelle: eigene Darstellung.

\section{Perspektiven für Unternehmen und Wirtschaftspolitik}

Der digitale Zwilling ermöglicht es, eine Plattform mit hoher monopolitischer Macht im Sinne des Konzepts zweiseitiger Märkte aufzubauen (Rochet und Tirole, 2003). Sobald eine kritische Grenze durchstoßen wird, ${ }^{16}$ werden die Netzwerkeffekte so profitabel (Evans und Schmalensee, 2010), dass die Kontrollkosten nach den PrinzipalAgent-Überlegungen grundlegend $\mathrm{zu}$ bewältigen sind und damit Wachstumsperspektiven nach oben offen werden, was beispielsweise Internetgiganten wie Amazon, Google oder Tencent zeigen. Besonders das Dominieren der Standards für die Beschreibung dürfte wirtschaftspolitisch von nationaler Bedeutung sein. Damit kann Marktmacht entstehen, die die Wettbewerbsaufsicht interessieren muss (Evans, 2003); es kann sich aber auch ein nationaler Wettbewerbsvorteil ergeben.

Schließlich stehen auch viele forschungsintensiv, auf Basis neuer Werkstoffe erzeugte Produkte vor dem Problem, dass sich der Produktlebenszyklus der Vermarktungsphase stetig verkürzt, hingegen sich die Zeit zur Marktreife (TtM, Time to Market) verlängert. Eine Möglichkeit, den digitalen Zwilling zu nutzen und beide Phasen stärker als bisher durch Simulation und Szenariobildung zu synchronisieren, also TDM, wird Ökonomie und Technologie verklammern. Dies ermöglicht ein Verstetigen der Innovation und den Aufstieg zum "industriellen Führer", der die Länge des Produktlebenszyklus festlegt.

16 Der Nutzen eines Netzwerks wächst üblicherweise in quadratischer Folge mit der Zahl der Benutzer, wohingegen die Stückkosten sinken, weil hohe Fixkosten auf eine wachsende Zahl von Benutzern verteilt werden. 
Aus staatlicher Sicht stellt sich die ordnungsökonomische Frage nach dem wettbewerblichen Rahmen, da Plattformökonomien förmlich dazu einladen, diese TDM-Märkte mit ihrer hohen Informationsbasis zu beherrschen. Denn dem Vorteil eine effizienten Lieferkettendurchdringung - auch als Unterstützung von Compliance-Regelungen - steht der Nachteil von Marktmacht und damit auch Unbestreitbarkeit gegenüber. Gerade die aktuelle Diskussion um die Internetgiganten verdeutlich den Handlungsbedarf, auch im Hinblick auf ein Abwägen der Vorteile der internationalen Arbeitsteilung und damit der Verflechtung der Wertschöpfungsketten - einschließlich der Recyclingketten - gegen das, was mit dem Begriff der Technologiesouveränität beschrieben ist.

\section{Literatur}

Acatech (2007), acatech diskutiert, Werkstoffe als Motor für Innovationen, Hartwig Höcker (Hrsg.), acatech workshop, Berlin, 17. Oktober.

Blum, U., T. Bahke und G. Eickhoff (2002), Normen und Wettbewerb, Beuth Verlag.

Blum, U. (2018), Der Kampf um Wertschöpfungsketten - Krieg gegen den Freihandel?, Wirtschaftsdienst, 98(10), 737-743.

Blum, U. (2020), Wirtschaftskrieg - Rivalität ökonomisch zu Ende denken, Springer.

Blum, U. und P. Boroskikh (2021), Mehrstufiges Recycling im System des Total Design Management: Das Zwiebelmodell, in U. Blum und R. W. Wehrspohn (Hrsg), Substitution, Recycling, Design und Nachhaltigkeit: Neue Wege der Werkstoffökonomik, Series in Political Economy and Economic Governance, 15.

Bocken, N. M. P., I. de Pauw, C. Bakker und B van der Grinten (2016), Product Design and Business Model Strategies for a Circular Economy, Journal of Industrial and Production Engineering, 33(5), 308-320.

Büttner, P. und J. Gutzmer (2017), Re-Mining: Ressourcen aus Bergbauhalden, 248, in U. Blum (Hrsg.), Die Nutzung von Ressourcenknappheit - mehr als eine ökonomische Fragestellung, Series in Political ECOnomy and Economic Governance, 10, 213-225.

Cludius, J., H. Hermann, F. C. Matthes und V. Graichen (2014), The Merit Order Effect of Wind and Photovoltaic Electricity Generation in Germany 2008-2016: Estimation and Distributional Effects, Energy Economics, 44, Juli, 302-313.

Coase, R. H. (1937), The Nature of the Firm, Economica, 4, 386-406.

Cossu, R. und I. D. Williams (2015), Urban Mining, Concepts, Terminology, Challenges, Waste Management, 45, 1-3.

Dietz, S. (2021), Designer tragen eine große Verantwortung (Interview), Magazin der Frankfurter Allgemeinen Zeitung, Januar, 30-32.

Ettenson, R., J. Krogstad und J. Shanteau (1987), Expert Judgment: Is More Information Better?, Psychology Reports, 60, 227-238.

EU (2020), Circular Economy Action Plan, https://ec.europa.eu/environment/circular-economy/ (22. Februar 2021).

Evans, D. S. (2003), The Antitrust Economics of Multi-Sided Platform Markets, Yale Journal on Regulation, 20(2), 325-382.
Evans, D. S. und R. Schmalensee (2010), Failure to Launch: Critical Mass in Platform Businesses, Review of Network Economics, 9(4), 1-26.

Fraunhofer-Verbund Materials, Materials Data Space ${ }^{\circledR}$ - Der Werkstoff wird digital, https://www.fraunhofer-materials-data-space.de/.

Greenhut, M., G. Norman und L.-S. Hung (1987), The Economics of Imperfect Competition: a Spatial Approach, Cambridge University Press.

Hoenderdaal, S., L. Tercero-Espinoza, F. Maerscheider-Weidemann und W. Graus (2013), Can a Dysprosium Shortage Threaten Green Energy Technologies?, Energy, 49, 344-355.

IMWS (2018), Sachsen-Anhalt beschließt Förderung für Fraunhofer-Pilotanlage CARBONTRANS in Leuna, 18. April, https://www.imws.fraunhofer.de/de/presse/pressemitteilungen/pilotanlage-carbontrans.html (16. Februar 2021).

Kahneman, D. und T. Tversky (1979), Prospect Theory: An Analysis of Decision under Risk, Econometrica, 47(2), 263-291.

Kerr, R. A. (2015), Wann wird Kupfer knapp, Spektrum der Wissenschaft, 1, 14-17.

Low, J. S. C., W. F. Lu und B. Song (2014), Methodology for an Integrated Life Cycle Approach to Design for Environment, Key Engineering Materials, 572, 20-23.

Perey, R., S. Benn, R. Agarwal und M. Edwards (2018), The place of waste: Changing business value for the circular economy, Business Strategy and the Environment, 27(5), 631-642.

Rajan, K. (2015), Materials Informatics: The Materials "Gene" and Big Data, Annual Review of Materials Research, 45, 153-169.

Ramakrishna, S., T.-Y. Zhang, W.-C. Lu, Q. Qian, J. S. C. Low, J. H. R. Yune, D. Z. L. Tan, S. Bressan, S. Sanvito und S. R. Kalidini (2018), Materials Informatics, Journal of Intelligent Manufacturing.

Reuter, M. A., A. v. Schaick und M. Ballester (2018), Limits of the Circular Economy, Fairphone Modular Design Pushing the Limits, World of Metals - ERZMETALL, 71(2), 68-79.

Rochet, J. C. und J. Tirole (2003), Platform Competition in Two-sided Markets, Journal of the European Economic Association, 1(4), 990-1029.

Schleich, B., N. Anwer, L. Mathieu und S. Wartzack (2017), Shaping the digital twin for design and production engineering, CIRP Annals Manufacturing Technology, 66/1, https://hal.archives-ouvertes.fr/hal01513846 (24. September 2019).

Sensfuß, F., M. Ragwitz und M. Genoese (2008), The Merit-Order Effect: a Detailed Analysis of the Price Effect of Renewable Electricity Generation in Spot Market Prices in Germany, Energy Policy, 36(8), 3086-3094.

Tseng, M. L., R. R. Tan, A. S. F. Chiub, C.-F. Chien und T. C. Kuo (2018), Circular Economy Meets Industry 4.0: Can Big Data Drive Industrial Symbiosis?, Resources, Conservation \& Recycling, 131, 146-147.

VDI (2014), Studie Werkstoffinnovationen für nachhaltige Mobilität und Energieversorgung.

Vidal, O., B. Goffé und N. Arndt (2013), Metals for a Low-Carbon Society, Nature Geoscience, 6, 894-896.

Wang, Z., X. Yang, Y. Zheng, Q. Yong, H. Su und C. Yang (2014), Integrated Materials Design and Informatics Platform, within the Materials Genome Framework, China Science Bulletin, 59(15), 1755-1764.

Wehrspohn, R. B., W.-G. Drossel und R. Stauber (2018), Kritikalität seltener Erden, Fraunhofer Gesellschaft, Abschlussbericht, V, 370.

Williamson, O. E. (1985), The Economic Institutions of Capitalism: Firms, Markets, Relational Contracting, The Free Press.

Williamson, O. (2002), The Theory of the Firm as Governance Structure: From Choice to Contract, Journal of Economic Perspectives, 16(3), 171-195.

Wuppertal Institut für Klima, Umwelt, Energie (2013), 18 Factsheets zum Thema Mobiltelefone und Nachhaltigkeit.

Title: Recycling of Materials, Components and Products: An Economic Challenge

Abstract: In view of the earth's limited landfill capacity and the increasing criticality of resources and supply chains, the recycling of the material flows is of central importance. Waste becomes a resource. Knowledge of its material composition becomes the central starting point for the control of eco-economically efficient value cycles. The digital mirroring of real processes in the sense of Industry 4.0 makes this possible. The concept of Total Design Management presented here enables the simultaneous optimisation of material, product and recycling design. Sustainability is strictly defined in relation to the system boundaries given by the digital twin. From an economic perspective, there is an economically justifiable trade-off between international division of labour and technology sovereignty. How this ultimately turns out depends heavily on the regulation of the markets and the supply chain risks.

JEL Classification: D9, L12, M11, Q20 